\title{
Determination of tungsten and molybdenum concentrations from an X-ray range spectrum in JET with the ITER-Like Wall configuration
}

\author{
T. Nakano ${ }^{1}$, A. E. Shumack ${ }^{2,3}$, C. F. Maggi ${ }^{2}$, M. Reinke ${ }^{4}$, K. D. \\ Lawson $^{2}$, I. Coffey ${ }^{2}$, T. Pütterich ${ }^{5}$, S. Brezinsek ${ }^{6}$, B. Lipschultz ${ }^{4}$, G. F.

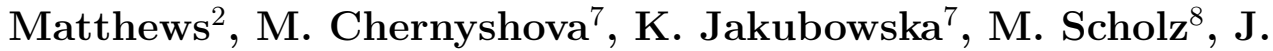 \\ Rzadkiewicz $^{7,9}$, T. Czarski ${ }^{7}$, W. Dominik ${ }^{10}$, G. Kasprowicz ${ }^{11}$, K. \\ Pozniak $^{11}$, W. Zabolotny ${ }^{11}$, K.-D.Zastrow ${ }^{2}$, N. J. Conway ${ }^{2}$ and JET \\ contributors*
}

EUROfusion consortium, JET, Culham Science Centre, Abingdon, OX14 3DB, UK

1 Japan Atomic Energy Agency, 801-1, Naka, Ibaraki, 311-0193, Japan

2 CCFE, Culham Science Centre, Abingdon, Oxon, OX14 3DB, UK

${ }^{3}$ FOM Institute DIFFER, Edisonbaan 14, NL-3439 MN, Nieuwegein, The Netherlands

${ }^{4}$ University of York, Heslington, YO10 5DD, UK

${ }^{5}$ Max-Planck-Institut für Plasmaphysik, 85748 Garching, Germany

${ }^{6}$ Association Forschungszentrum Jülich GmbH 52425 Jülich Germany

${ }^{7}$ Instiute of Plasma Physics and Laser Microfusion, Hery 23, 01-497 Warsaw, Poland

${ }^{8}$ Institute of Nuclear Physics PAN ul. Radzikowskiego 152 31-342 Kraków, Poland

${ }^{9}$ Narodowe Centrum Badań Jạdrowych ul. Andrzeja Sołtana 7 05-400 Otwock,Świerk, Poland

${ }^{10}$ Warsaw University, Faculty of Physics, Institute of Experimental Physics, 00-681 Warsaw, Poland

${ }^{11}$ Warsaw University of Technology, Institute of Electronic Systems, 00-665 Warsaw, Poland

* See the Appendix of F. Romanelli et al., Proc. of the 25th IAEA FEC 2014, St. Petersburg, Russia

E-mail: nakano.tomohide@jaea.go.jp 


\begin{abstract}
The $\mathrm{W}^{45+}$ and $\mathrm{W}^{46+} 3 \mathrm{p}-4 \mathrm{~d}$ inner shell excitation lines in addition to $\mathrm{Mo}^{32+} 2 \mathrm{p}-$ 3s lines have been identified from the spectrum taken by an upgraded high-resolution X-ray spectrometer. It is found from analysis of the absolute intensities of the $\mathrm{W}^{46+}$ and $\mathrm{Mo}^{32+}$ lines that $\mathrm{W}$ and Mo concentrations are in the range of $\sim 10^{-5}$ and $\sim 10^{-6}$, respectively, with a ratio of $\sim 5 \%$ in JET with the ITER-Like Wall configuration for ELMy H-mode plasmas with a plasma current of 2.0- 2.5 MA, a toroidal magnetic field of $2.7 \mathrm{~T}$ and a neutral beam injection power of 14 - $18 \mathrm{MW}$. For the purpose of checking self-consistency, it is confirmed that the W concentration determined from the $\mathrm{W}^{45+}$ line is in agreement with that from the $\mathrm{W}^{46+}$ line within $20 \%$ and that the plasma effective charge determined from the continuum of the first order reflection spectrum is also in agreement with that from the second order within $50 \%$. Further, the determined plasma effective charge is in agreement with that determined from a visible spectroscopy, confirming that the sensitivity of the X-ray spectrometer is valid and that the $\mathrm{W}$ and the Mo concentrations are also likely to be valid.
\end{abstract}




\section{Introduction}

It has been decided that ITER will be operated from day one with tungsten (W) divertor and beryllium (Be) first wall $[1,2]$ mainly in order to reduce tritium retention inside the vacuum vessel. In JET, in order to provide the physics and engineering basis for the exploitation of ITER, the original carbon plasma-facing components were all replaced by tungsten ones for the divertor and beryllium ones for the main chamber. JET with this new ITER-like wall configuration (hereafter, JET-ILW) has been in operation since $2011[3,4]$. In such a wall configuration, one of the issues is impact of tungsten on plasma fusion performance. Hence quantitative $\mathrm{W}$ concentration monitoring is one of the important research objects in the JET-ILW project.

In parallel to JET modification into JET-ILW, an existing high-resolution X-ray crystal spectrometer was upgraded for the purpose of $\mathrm{W}$ concentration monitoring. This X-ray spectrometer was originally prepared to measure ion temperature [5] and toroidal plasma rotation [6] from, respectively, Doppler broadening and shift of the spectral line of He-like $\mathrm{Ni}$ ion $\left(\mathrm{Ni}^{26+}\right)$ in addition to $\mathrm{Ni}$ concentration. The X-ray spectrometer has now also started monitoring $\mathrm{W}$ concentration since a second crystal and detector for W were installed [7-9]. Because the sensitivity of the X-ray spectrometer is given as a photon throughput [9], it is possible to determine the $\mathrm{W}$ concentration from the absolute intensity of $\mathrm{W}$ lines.

To determine $\mathrm{W}$ concentration in plasmas with an electron temperature of $\sim 5 \mathrm{keV}$, where a dominant $\mathrm{W}$ charge state is around 46 , the $\mathrm{W}^{45+}(4 \mathrm{~s}-4 \mathrm{p}: \lambda=6.2 \mathrm{~nm})$ spectral line has been often used [10-12]. One advantage of this line is that it is exceptionally isolated from other spectral lines in terms of wavelength even with $\Delta n=0$ transition $(n$ : principal quantum number), although many $\mathrm{W}$ spectral lines with $\Delta n=0$ transitions with charge states up to 45 fall into $4-7 \mathrm{~nm}$ and blend each other, forming a W line complex. This results in difficulties in identifying transitions and defining line intensities, which are essential information for determining $W$ concentration. Except for the $\mathrm{W}^{45+}$ spectral line, it is difficult to find an isolated spectral line in the vacuum ultra violet range unless a very high-resolution spectrometer is used. The upgraded Xray spectrometer offers another opportunity to measure isolated $\mathrm{W}$ spectral lines. The $\mathrm{X}$-ray spectrometer has sufficiently high wavelength resolution so that $\mathrm{W}$ spectral lines can be measured without line blends, and that further Doppler broadening and shift of the $\mathrm{W}$ spectral lines can be measured.

This paper describes line identification, comparison of $\mathrm{W}$ concentrations determined from the line intensities of two different charge states of $\mathrm{W}$ ions measured simultaneously with the same diagnostic and comparison of plasma effective charge determined from continuum intensities at two different photon energies, in order to confirm self-consistency within the X-ray spectrometer. Additionally, comparison of the W concentration with the Mo concentration is mentioned because Mo spectral lines are coincidentally found in the same wavelength range as the $\mathrm{W}$ spectral lines. The present report provides the first data on $\mathrm{W}$ concentrations since the upgrade of the X-ray 
spectrometer.

\section{Experimental}

\subsection{Spectrometer setup}

The purpose of the upgrade of the high-resolution X-ray spectrometer is to measure the $\mathrm{W}^{46+} 3 \mathrm{p}-4 \mathrm{~d}$ line at $0.52004 \mathrm{~nm}$ (the wavelength from the on-line database [13]) to determine $\mathrm{W}$ concentrations while the original $\mathrm{Ni}$ concentration measurement is still kept available. Figure 1 (a) shows a bird's eye view of the X-ray spectrometer. The $\mathrm{X}$-ray spectrometer is built in Johann mounting with a Rowland circle radius of 12.5 $\mathrm{m}$ [5]. X-ray emissions from the plasma pass through a 300- $\mu \mathrm{m}$-thick Be window at the vacuum vessel and a 20-m-long beam line, and then inject a cylindrically bent $\mathrm{SiO}_{2}$ crystal (1011) $(2 d=0.668 \mathrm{~nm}, d$ : lattice spacing) with a width of $230 \mathrm{~mm}$ and a height of $35 \mathrm{~mm}$ for the $\mathrm{W}$ channel. At the crystal, the injected X-ray is dispersed at a Bragg angle of $51^{\circ}$ and then detected by a new Gas Electron Multiplier (GEM) detector $[7-9,14,15]$ with a width of $205 \mathrm{~mm}(256$ strips $\times 0.8 \mathrm{~mm})$ at a temporal resolution of $10 \mathrm{~ms}$. Because each strip is energy sensitive, the second order reflection spectrum in addition to the first order reflection spectrum can be measured at the same time. As shown in Figs. 1 (b) and (c), the diagnostic line-of-sight is on the mid-plane of the torus, $0.2 \mathrm{~m}$ below the magnetic axis of typical diverted plasmas, and the line-ofsight is in the opposite direction to the plasma current. Thus, in the case of co-rotating plasmas, blue-shifted spectrum is observed due to Doppler shift.

The measurable spectral band in the first order of reflection is $0.0043 \mathrm{~nm}$ at a wavelength of $0.52 \mathrm{~nm}$, or a photon energy of $\sim 2.4 \mathrm{keV}$. By wavelength-scanning, $0.502-0.536 \mathrm{~nm}$ can be measured. An inverse linear dispersion is $2.1 \times 10^{-5} \mathrm{~nm} / \mathrm{mm}$. The sensitivity is calculated as a product of photon-throughputs at each component of the spectrometer: X-ray reflectivity of the crystal, transmittance of a $300-\mu \mathrm{m}$-thick Be window at the torus, a 9-cm-long He gas buffer in front of the detector, a 5- $\mu$ m-thick Mylar window at the detector, resulting in $1.6 \times 10^{-11}$ [counts $\mathrm{ph}^{-1} \mathrm{~m}^{2} \mathrm{sr}$ ] for the first order reflection spectrum and $2.9 \times 10^{-11}$ [counts $\left.\mathrm{ph}^{-1} \mathrm{~m}^{2} \mathrm{sr}\right]$ for the second one at $\sim 4.8$ $\mathrm{keV}[9]$.

Figure 1 (c) also shows the line-of-sight of a high-resolution Thomson scattering (HRTS) system. The HRTS system measures spatial profiles of electron temperature and density along the line-of-sight with a spatial resolution of $16 \mathrm{~mm}$ at the plasma core [16]. These are used to calculate spatial profiles of the $\mathrm{W}$ and Mo emissions, which depend on electron temperature and density. In addition, the line-of-sight of a visible bremsstrahlung emission measurement, which is used for determination of a plasma effective charge, is shown. 


\subsection{Measurement}

An X-ray spectrum analysed for line identification is taken in an ELMy H-mode discharge with a plasma current of 2.0 MA and a toroidal magnetic field of $2.7 \mathrm{~T}$. Traces of the parameters of this discharge are shown in Fig. 2; plasma heating is performed by a neutral beam (NB) with an injection power of $14 \mathrm{MW}$. During the steady state NB injection, the radiation power from bolometry in the main plasma is about $3 \mathrm{MW}$. From the radiation power signal in the divertor plasma, regular ELM activities at a frequency of $47 \mathrm{~Hz}$ are seen. The $\mathrm{D}_{2}$ puff rate is modulated at $3 \mathrm{~Hz}$ between $1.2 \times 10^{22} \mathrm{D} / \mathrm{s}$ and $3.0 \times 10^{22} \mathrm{D} / \mathrm{s}$, although the modulation does not play a role for the line identification. The electron temperature at the plasma core measured by the HRTS system is $4 \mathrm{keV}$ and the electron density $6 \times 10^{19} \mathrm{~m}^{-3}$. At $18.0 \mathrm{~s}$, molybdenum (Mo) is injected by laser ablation (Laser-blow-off, LBO) [17] in order to confirm identification of Mo lines. As shown in Fig. 3, the intensities of two of the central spectral lines increase rapidly after the Mo injection, while those are lower than the spectral line at a strip number of 40 before the Mo injection. This confirms that the two spectral lines are emitted from Mo ions while the others not from Mo ions.

Figure 4 (a) shows time-averaged spectra between $13.0 \mathrm{~s}$ and $15.0 \mathrm{~s}$ taken in the discharge shown in Fig. 2. As already shown in Fig. 3, five spectral lines, two of which are confirmed to be Mo lines, are seen in the first order reflection spectrum. This is used for detailed analysis for line identification, later. In contrast, no spectral lines are seen in the second order reflection spectrum. This feature is desirable for analyses of the continuum intensity, for example, to determine a plasma effective charge $\left(Z_{\text {eff }}\right)$.

\section{Analysis}

\subsection{Spectrum analysis for line identification}

One of the difficulties in high resolution spectroscopy is line identification. This is due to the difficulty in determining the wavelength; slight misalignment of the instrument results in large uncertainties in wavelength and also known lines rarely fall in a very narrow spectral band, making the experimental wavelength calibration uncertain. To overcome these drawbacks, we employed the following two methods: i) Mo laser-blowoff experiment and ii) validation of the modelled spectrum using Flexible Atomic Code (FAC) [18], by comparing it with published spectra [19].

As already shown in Fig. 3, the Mo laser-blow-off experiment confirms that two of the central lines are from Mo ions. However, charge states and transitions are not known yet only from this laser-blow-off experiment.

In order to identify the observed spectral lines, atomic structure calculation is performed for $\mathrm{W}$ and Mo ions by FAC, which adopts the full-relativistic DiracFock-Slater iteration method with configuration interactions for calculation of atomic structures and the distorted wave approximation for calculations of electron-impact cross sections. For example, the following electron configurations are considered for $\mathrm{W}^{46+}$, 
Ni-like W ion;

$$
\begin{array}{lll}
3 \mathrm{p}^{6} & 3 \mathrm{~d}^{10} & \text { : ground state, Ni-like closed shell structure } \\
3 \mathrm{p}^{6} & 3 \mathrm{~d}^{9} 4 l & : \text { singly excited state } \\
3 \mathrm{p}^{6} & 3 \mathrm{~d}^{9} 5 l & : \text { singly excited state } \\
3 \mathrm{p}^{5} & 3 \mathrm{~d}^{10} 4 l & \text { : inner shell }(3 \mathrm{p}) \text { excited state } \\
3 \mathrm{p}^{5} & 3 \mathrm{~d}^{10} 5 l & : \text { inner shell }(3 \mathrm{p}) \text { excited state } \\
3 \mathrm{p}^{6} & 3 \mathrm{~d}^{8} 4 l 4 l^{\prime} & : \text { doubly excited state } \\
3 \mathrm{p}^{5} & 3 \mathrm{~d}^{9} 4 l \quad 4 l^{\prime} & : \text { doubly and inner shell }(3 \mathrm{p}) \text { excited state }
\end{array}
$$

, where $l$ and $l^{\prime}$ are azimuthal quantum numbers. The atomic structure calculation gives about 6000 energy levels. Spontaneous transition rates and excitation/deexcitation rate coefficients between all the energy levels and ionization rate coefficients from all the energy levels to the $\mathrm{W}^{47+}$ ground level are calculated, and then used for collisionalradiative modelling to calculate $\mathrm{W}^{46+}$ spectra.

Although similar calculations are performed for $\mathrm{W}^{25+}-\mathrm{W}^{71+}$, it is probable that predominant contributors are $\mathrm{W}^{4+}-\mathrm{W}^{46+}$. This is because the ionization potentials of $\mathrm{W}^{q+}(q \leq 43)$ are lower than the measurable photon energy range of the $\mathrm{X}$ ray spectrometer $(\sim 2.4 \mathrm{keV})$. For example, the ionization potential of $\mathrm{W}^{43+}$ is 2.2 $\mathrm{keV}$. Hence, no photons emitted from levels below the ionization potential fall in the measurable photon energy range of the X-ray spectrometer, except for photons from levels above the ionization potential, for example, auto-ionization levels. But emission intensities from the auto-ionization levels should be very low, compared to those from singly excited levels below the ionization potential. In addition, the ionization potential of $\mathrm{W}^{46+}(4.1 \mathrm{keV})$ jumps from that of $\mathrm{W}^{45+}(2.4 \mathrm{keV})$ due to the Ni-like closed shell structure. Because of this jump, $\mathrm{W}^{46+}$ is more difficult to ionize into $\mathrm{W}^{47+}$ while $\mathrm{W}^{45+}$ easier to ionize into $\mathrm{W}^{46+}$ at the central electron temperature of the discharge $(\sim 4 \mathrm{keV})$. Hence, the $\mathrm{W}^{46+}$ population tends to be high compared to $\mathrm{W}^{q+}(q \geq 47)$. Therefore, for the above two reasons, the main focus of the calculation is on $\mathrm{W}^{44+}-\mathrm{W}^{46+}$. As for Mo ions, by similar reasons, a predominant contributor to the spectrum of the X-ray spectrometer is $\mathrm{Mo}^{32+}$.

First, the calculated spectra are compared to published spectra [19], which cover the spectral range of the X-ray spectrometer. This comparison results in good agreement within the spectral resolution of the published spectra, which is much lower than that

of the X-ray spectrometer. The calculated spectrum for $\mathrm{W}^{45+}, \mathrm{W}^{46+}$ and $\mathrm{Mo}^{32+}$ at a density ratio of 1.0: 0.3 : 0.7 and an electron temperature of $4 \mathrm{keV}$ is shown in Fig. 4 (b). This spectrum is compared to the measured spectrum.

\subsection{Line identification}

Figure 4 compares the experimental spectrum with the calculated spectrum, showing overall agreement; as already described in Sec.2.2, the Mo injection experiment confirmed that the two central lines were from Mo ions. These lines were well reproduced 
by the calculated spectrum and both identified as $\mathrm{Mo}^{32+}(2 \mathrm{~s}-3 \mathrm{p})$ lines. More exactly, from the shorter wavelength, the following two lines were identified:

- $\mathrm{Mo}^{32+}\left(2 \mathrm{p}^{6}{ }^{1} \mathrm{~S}_{0}-2 \mathrm{p}^{5} 3 \mathrm{~s}^{3} \mathrm{P}_{1}: \lambda=0.52069 \mathrm{~nm}[13]\right.$, electric dipole (E1) transition)

- $\mathrm{Mo}^{32+}\left(2 \mathrm{p}^{6}{ }^{1} \mathrm{~S}_{0}-2 \mathrm{p}^{5} 3 \mathrm{~s}^{3} \mathrm{P}_{2}: \lambda\right.$, not shown in Ref. [13], magnetic quadrupole (M2) transition)

These two lines were already identified in Alcator C [20] and FTU tokamaks [21]. Recently, theoretical treatment for the reason that the M2 line intensity, which was thought to be very weak, was comparable to the E1 line intensity was given. The theory indicated that the M2 line was induced by a magnetic field, suggesting a possibility to diagnose the magnetic field strength [22]. Implementation of this effect, which was not included in the present calculation, may give a better agreement with the measured M2 intensity because the present measurement was performed under a magnetic field of 2.7 $\mathrm{T}$ at the magnetic axis. The remaining lines were well reproduced by the calculated spectrum, resulting in the following identification:

- $\mathrm{W}^{46+}\left(3 \mathrm{p}^{6} 3 \mathrm{~d}^{10}{ }^{1} \mathrm{~S}_{0}-3 \mathrm{p}^{5} 3 \mathrm{~d}^{10} 4 \mathrm{~d}(3 / 2,5 / 2)_{1}^{o}: \lambda=0.52004 \mathrm{~nm}[13]\right)$

- $\mathrm{W}^{45+}\left(3 \mathrm{p}^{6} 3 \mathrm{~d}^{10} 4 \mathrm{~s}^{2} \mathrm{~S}_{1 / 2}-3 \mathrm{p}^{5} 3 \mathrm{~d}^{10} 4 \mathrm{~s} 4 \mathrm{~d}(3 / 2,2)_{3 / 2}^{o}: \lambda\right.$, not shown in Ref. [13]) )

- $\mathrm{W}^{45+}\left(3 \mathrm{p}^{6} 3 \mathrm{~d}^{10} 4 \mathrm{~s}^{2} \mathrm{~S}_{1 / 2}-3 \mathrm{p}^{5} 3 \mathrm{~d}^{10} 4 \mathrm{~s} 4 \mathrm{~d}(3 / 2,2)_{1 / 2}^{o}: \lambda=0.52289 \mathrm{~nm}[13]\right)$

- $\mathrm{W}^{45+}\left(3 \mathrm{p}^{6} 3 \mathrm{~d}^{10} 4 \mathrm{~s}^{2} \mathrm{~S}_{1 / 2}-3 \mathrm{p}^{5} 3 \mathrm{~d}^{10} 4 \mathrm{~s} 4 \mathrm{~d}(3 / 2,3)_{3 / 2}^{o}: \lambda=0.52379 \mathrm{~nm}[13]\right)$

These transitions were due to inner shell excitation from $3 \mathrm{p}$ to $4 \mathrm{~d}$ electron, which required a high excitation energy close to the ionization potential of $\mathrm{W}^{45+}, 2.41 \mathrm{keV}$.

Note that the spectrum is not wavelength-calibrated and that the measured spectrum should be blue-shifted due to the Doppler shift while the calculated spectrum is plotted as calculated by FAC, or by stationary wavelength. Thus, the correspondence between the strip number and the wavelength, shown respectively at the top of Fig. 4 (a) and the bottom of Fig. 4 (b), always depends on the Doppler shift, or plasma toroidal rotation. This is the reason why the $\mathrm{Mo}^{32+}$ lines are shifted toward the longer wavelength; generally, plasma toroidal rotation speed is the highest at the plasma core and decreases with increasing plasma minor radius. As shown in Fig. 5 later, the $\mathrm{W}^{45+}$ and $\mathrm{W}^{46+}$ emissions are from the plasma core while the $\mathrm{Mo}^{32+}$ emission covers a broader volume, extending radially outwards. This results in smaller Doppler shift for $\mathrm{Mo}^{32+}$ lines.

\subsection{Intensity analysis for $W$ and $M o$ concentrations}

From the measured $\mathrm{W}^{46+}$ line intensity, the $\mathrm{W}$ concentration, $c_{\mathrm{W}}=n_{\mathrm{W}} / n_{\mathrm{e}}\left(n_{\mathrm{W}}\right.$ : tungsten density and $n_{\mathrm{e}}$ : electron density) is determined from the following equation on the assumption of uniform $\mathrm{W}$ density profile for the region where the $\mathrm{W}^{46+}$ line is emitted (a similar equation for the $\mathrm{Mo}^{32+}$ line intensity to determine the Mo concentration, $\left.c_{\mathrm{Mo}}=n_{\mathrm{Mo}} / n_{\mathrm{e}}\right)$ :

$$
n_{\mathrm{W}}=\frac{I^{\mathrm{W}^{46+}}}{\int \mathrm{FA}^{\mathrm{W}^{46+}}\left(R_{\mathrm{LOS}}\right) \mathrm{PEC}^{\mathrm{W}^{46+}}\left(R_{\mathrm{LOS}}\right) n_{\mathrm{e}}\left(R_{\mathrm{LOS}}\right) d R_{\mathrm{LOS}}},
$$




$$
c_{\mathrm{W}}=n_{\mathrm{W}} / n_{\mathrm{e}}=\frac{I^{\mathrm{W}^{46+}}}{\int \mathrm{FA}^{\mathrm{W}^{46+}}\left(R_{\mathrm{LOS}}\right) \mathrm{PEC}^{\mathrm{W}^{46+}}\left(R_{\mathrm{LOS}}\right) n_{\mathrm{e}}\left(R_{\mathrm{LOS}}\right)^{2} d R_{\mathrm{LOS}}},
$$

where $I^{\mathrm{W}^{46+}}\left[\mathrm{ph} \mathrm{m}{ }^{-2} \mathrm{~s}^{-1}\right]$ is the measured $\mathrm{W}^{46+}$ line intensity, $R_{\mathrm{LOS}}[\mathrm{m}]$ the major radius along the line-of-sight of the X-ray spectrometer, $\mathrm{FA}^{\mathrm{W}^{46+}}\left(=n_{\mathrm{W}^{46+}} / n_{\mathrm{W}}\right)$ the fractional abundance of $\mathrm{W}^{46+}, \mathrm{PEC}^{\mathrm{W}^{46+}}\left[\mathrm{ph} \mathrm{m}^{3} \mathrm{~s}^{-1}\right]$ the photon emission coefficient for the measured $I^{\mathrm{W}^{46+}}$ line.

In order to evaluate the $\mathrm{W}$ concentration $c_{\mathrm{W}}, \mathrm{FA}^{\mathrm{W}^{46+}}$ and $\mathrm{PEC}^{\mathrm{W}^{46+}}$ need to be evaluated as a first step. Given that $\mathrm{FA}^{\mathrm{W}^{46+}}$ and $\mathrm{PEC}^{\mathrm{W}^{46+}}$ are calculated with electron temperature and density, they can be plotted as a function of a normalised poloidal magnetic flux $\psi_{\text {pol }}$ by using electron temperature and density profiles over $\psi_{\text {pol }}$. Figure 5 (a) shows measured electron temperature and density profiles with fitting curves (only $\psi_{\text {pol }} \leq 0.9$ ) as a function of $\psi_{\text {pol }}$. From the electron temperature and density profiles, $\mathrm{W}^{45+}, \mathrm{W}^{46+}$ and $\mathrm{Mo}^{32+}$ fractional abundance under ionization equilibrium are calculated with ADAS ionization/recombination rates [23] for $\mathrm{W}$ ion and with the data in Ref. [24] for Mo ions. As shown in Fig. 5 (b), the $\mathrm{W}^{45+}$ and $\mathrm{W}^{46+}$ fractional abundance increase toward the plasma core while $\mathrm{Mo}^{32+}$ fractional abundance is approximately constant within $\psi_{\text {pol }}<0.4$ and is slightly hollow at the centre. Note that the fractional abundances are not calculated at $\psi_{\text {pol }} \leq 0.05$ because the line-of-sight of the $\mathrm{X}$ ray spectrometer does not pass through this range. Photon emission coefficients are calculated by FAC collisional-radiative modeling [18] also using the measured electron temperature and density profiles shown in Fig 5 (a). Figure 5 (c) shows the calculated photon emission coefficients. The $\mathrm{W}^{45+}, \mathrm{W}^{46+}$ and $\mathrm{Mo}^{32+}$ photon emission coefficients monotonically increase toward the plasma core.

Next, a product of FA, PEC and $n_{\mathrm{e}}$ is calculated. As shown in Fig. 5 (d), the profile of the product is similar to that of the fractional abundance, FA, shown in Fig. 5 (b); the products for $\mathrm{W}^{45+}$ and $\mathrm{W}^{46+}$ increase toward the plasma core while that for $\mathrm{Mo}^{32+}$ is approximately constant within $\psi_{\text {pol }}<0.4$ and is slightly hollow at the centre.

Finally, the normalised poloidal magnetic flux $\psi_{\text {pol }}$ is converted to the major radius along the line-of-sight, $R_{\mathrm{LOS}}$, in order to evaluate line-integral emissivity along the lineof-sight. Figure 5 (e) shows the normalised poloidal magnetic flux $\psi_{\text {pol }}$ as a function of the major radius along the line-of-sight of the X-ray spectrometer, calculated from a magnetic equilibrium reconstructed by the EFIT code [25]. Given that the line-of-sight passes through the plasma between low- and high-field-side separatrix, the normalised poloidal magnetic flux is a downward-convex function of the major radius along the line-of-sight. Figure 5 (e) also shows the products of FA, PEC and $n_{\mathrm{e}}$ for $\mathrm{W}^{45+}, \mathrm{W}^{46+}$ and $\mathrm{Mo}^{32+}$. The product for $\mathrm{W}^{46+}$ has a peak at $R_{\mathrm{LOS}}$ of $\sim 3 \mathrm{~m}$. This is also the case for $\mathrm{W}^{45+}$. In contrast, the product for $\mathrm{Mo}^{32+}$ is approximately constant in an $R_{\mathrm{LOS}}$ range between 2.7 and $3.3 \mathrm{~m}$. The product is integrated along the line-of-sight as shown in eq.(1), and then, $\mathrm{W}$ density is evaluated. Similarly, the $\mathrm{W}$ concentration is evaluated from eq. (2). Because the $\mathrm{W}$ density and concentration can be determined from the measured $\mathrm{W}^{46+}$ intensity in addition to the measured $\mathrm{W}^{45+}$ intensity, they 
can be compared to each other. In contrast, this is impossible for the Mo density and concentration because spectral lines from only one charge state of Mo ion $\left(=\mathrm{Mo}^{32+}\right)$ is observed in the present measurement. The determined $\mathrm{W}$ and Mo concentrations are in the range of $10^{-5}$ and $10^{-6}$, respectively, as already shown in Fig. 2 (f), and the ratio of Mo to $\mathrm{W}$ concentration is $\sim 5 \%$.

\subsection{Continuum intensity analysis for $Z_{\text {eff }}$}

A plasma effective charge, $Z_{\text {eff }}$, is evaluated from the measured continuum emission. As already shown in Fig. 4 (a), clear continuum spectrum is observed in both the first and the second order spectra. In particular, the second order spectrum is better for the continuum spectrum analysis because no spectral lines are seen. In the case that the continuum spectrum is dominated by Bremsstrahlung emission, $\mathrm{Z}_{\text {eff }}$ can be evaluated with ease. However, it is possible that recombination continuum emission contributes to the measured continuum. Hence it is necessary to evaluate contributions of Bremsstrahlung and recombination continua to the measured continuum.

\subsubsection{Bremsstrahlung continuum}

As shown in Fig. 6, Bremsstrahlung continuum is emitted from a free electron, the final state of which is not bounded by plasma ions. Given that the photon energies for the first order spectrum are centred around $2.4 \mathrm{keV}$, only transitions with an energy difference of $\sim 2.4 \mathrm{keV}$ can contribute to the measured continuum intensity. The Bremsstrahlung continuum emissivity, $P^{\mathrm{B}}\left[\mathrm{Wm}^{-3}\right]$ is given by the following equation $[26]$ :

$$
\begin{aligned}
P^{\mathrm{B}}(\nu) d \nu & =n_{\mathrm{e}} n_{\mathrm{z}} z J\left(R / T_{\mathrm{e}}\right)^{0.5} \exp \left(-h \nu / T_{\mathrm{e}}\right) \frac{1}{2} g_{\mathrm{ff}} d \nu \\
\text { with } J & =\frac{2^{7}}{3^{1.5}}(2 \pi)^{0.5} \alpha\left(\frac{z^{2} R}{m c^{2}}\right)^{1.5} h c\left(a_{0} / z\right)^{2} \\
\text { and } Z_{\mathrm{eff}} & =\frac{\sum_{z} n_{\mathrm{z}} z^{2}}{n_{\mathrm{e}}}
\end{aligned}
$$

where $\nu$ indicates the photon frequency, $n_{\mathrm{z}}$ the impurity density with a charge $z, R$ the Rydberg constant $(=13.6 \mathrm{eV}), h$ the Planck's constant $\left(=6.6 \times 10^{-34} \mathrm{Js}\right), g_{\mathrm{ff}}$ the Gaunt factor [27], $\alpha$ the fine structure constant $(=1 / 137), m$ the electron mass $\left(=9.1 \times 10^{-31}\right.$ $\mathrm{kg}), c$ the speed of light $\left(=3.0 \times 10^{8} \mathrm{~ms}^{-1}\right)$, and $a_{0}$ the Bohr radius $\left(=5.3 \times 10^{-11} \mathrm{~m}\right)$. Similarly to determination of the $\mathrm{W}$ density , the line-integral Bremsstrahlung intensity is calculated with electron temperature and density profiles, and then a plasma effective charge $\mathrm{Z}_{\text {eff }}$ is evaluated from the measured continuum intensity.

\subsubsection{Recombination continuum}

As shown in Fig.6, recombination continuum is emitted from a free electron, the final level of which is bounded by plasma ions. Again, only transitions with energy difference between the initial and the final level of $\sim 2.4 \mathrm{keV}$ can contribute to the 
measured continuum intensity. Because the photon energy is a sum of the kinetic energy of the free electron and the energy difference between the bound level and the ionization potential, electrons with a kinetic energy of less than $2.4 \mathrm{keV}$ can contribute to the measured continuum intensity. For example, only electrons with a kinetic energy of $50 \mathrm{eV}$ can contribute to the measured continuum intensity in the case the electrons recombine directly to the ground level of $\mathrm{W}^{44+}$ given that the ionization potential of $\mathrm{W}^{44+}$ is $2.35 \mathrm{keV}$. In general formulation, the intensity of recombination continuum to an impurity ion with a charge of $\mathrm{q}$, for example $\mathrm{W}^{q+}, P_{q}^{\mathrm{R}}\left[\mathrm{Wm}^{-3}\right]$ is expressed with the following equation [26];

$$
\begin{aligned}
P_{q}^{\mathrm{R}}(\nu) d \nu & =\Sigma_{p} h \nu n_{\mathrm{W}^{q+}} n_{\mathrm{e}} f(\varepsilon) v \sigma_{\varepsilon, \mathrm{p}}(\varepsilon) d \varepsilon, \\
P^{\mathrm{R}}(\nu) d \nu & =\Sigma_{q} P_{q}^{\mathrm{R}}(\nu) d \nu,
\end{aligned}
$$

where $n_{\mathrm{W}^{q+}}$ indicates a $\mathrm{W}^{q+}$ density, $f(\varepsilon)$ an electron velocity distribution function, $v$ a electron velocity, $\sigma_{\varepsilon, \mathrm{p}}(\varepsilon)$ a recombination cross section of the electron with a kinetic energy of $\varepsilon$ to a bound level $p$ of $\mathrm{W}^{q+}$, and $P^{\mathrm{R}}(\nu) d \nu$ a total recombination continuum intensity. The recombination cross sections are calculated by FAC; recombination radiation to all the excited levels and the ground level, for example $\sim 6000$ levels for $\mathrm{W}^{46+}$, is considered for $\mathrm{W}^{26+}-\mathrm{W}^{71+}$. Similarly recombination radiation from $\mathrm{Mo}^{6+}$ $\mathrm{Mo}^{39+}$ is also considered. In the present study, Bremsstrahlung continuum intensity is evaluated by subtracting the calculated recombination continuum contribution from the measured continuum intensity, and then the plasma effective charge is evaluated.

\section{Results and discussion}

\subsection{W and Mo concentrations}

The $\mathrm{W}$ and Mo concentrations determined from the present analysis were already shown in Fig. 2 (f). The $\mathrm{W}$ and Mo concentrations were respectively in the range of $1 \times 10^{-5}$ and $1 \times 10^{-6}$. Here, in order to discuss spatial profiles of the $\mathrm{W}$ concentration, the $\mathrm{W}$ concentration determined from the $\mathrm{W}^{45+}$ intensity, $c_{\mathrm{W}}(45)$, was compared with that determined from the $\mathrm{W}^{46+}$ intensity, $c_{\mathrm{W}}(46)$. As shown in Fig. 7 (a), $c_{\mathrm{W}}(45)$ increased with increasing $c_{\mathrm{W}}(46)$. However, $c_{\mathrm{W}}(45)$ was systematically lower than $c_{\mathrm{W}}(46)$ by $\sim$ $20 \%$. This was also the case for data taken in different types of discharges: a nitrogen seeded ELMy H-mode discharge with a plasma current of 2.5 MA, a toroidal magnetic field of $2.7 \mathrm{~T}$, a neutral beam injection power of $18 \mathrm{MW}$, a central electron temperature of $4 \mathrm{keV}$, and a central electron density of $9 \times 10^{19} \mathrm{~m}^{-3}$ [28] and a non-seeded ELMy H-mode discharge with 2.5 MA, 2.7 T, $17 \mathrm{MW}, 3 \mathrm{keV}$ and $8 \times 10^{19} \mathrm{~m}^{-3}$ with the outer strike point on the horizontal target (bulk W target) [29].

There is a possibility that this systematic $20 \%$ deviation is due to the spatial profile of $\mathrm{W}$ concentrations; as shown in eq. $(2), c_{\mathrm{W}}(45)$ and $c_{\mathrm{W}}(46)$ reflect the $\mathrm{W}$ concentrations at the $\mathrm{W}^{45+}$ and the $\mathrm{W}^{46+}$ emission location, respectively. Given that the $\mathrm{W}^{46+}$ emission profile is slightly narrower than the $\mathrm{W}^{45+}$ emission profile as shown in Fig. $5(\mathrm{~d}), c_{\mathrm{W}}(46)$ reveals more central $\mathrm{W}$ concentration information than $c_{\mathrm{W}}(45)$. Hence, the difference 
between $c_{\mathrm{W}}(45)$ and $c_{\mathrm{W}}(46)$ suggests a possibility that the $\mathrm{W}$ concentration increases toward the plasma core. Because, however, difference of the emission profiles between $\mathrm{W}^{45+}$ and $\mathrm{W}^{46+}$ is small, it is difficult to investigate further details. To conclude this, sufficiently small uncertainty in atomic data is required. But still, it is possible to conclude that $c_{\mathrm{W}}(46)$ is in agreement with $c_{\mathrm{W}}(45)$ with $\sim 20 \%$ deviation, indicating self-consistency in the present analysis.

The Mo concentration $c_{\text {Mo }}$ increased very weakly with increasing $c_{\mathrm{W}}(46)$ as shown in Fig. 7 (b). In JET, no Mo materials are used for the plasma-facing components except for only one marker tile at the inner divertor [30]. One of the possible sources is $\mathrm{W}$ coated CFC tiles, which have a $\mathrm{W} / \mathrm{Mo} / \mathrm{W} / \mathrm{Mo}$ multilayer on a CFC substrate [31]. Once the top surface $\mathrm{W}$ coating is eroded or delaminated, the tile can be a Mo source. However, higher Mo concentration is found from the plasma with the outer strike point on the horizontal target, which is made of bulk tungsten, as shown in Fig. 7 (b). This indicates that the $\mathrm{W}$ coated tiles at the outer divertor are not necessarily the Mo source. Another possibilities for the Mo source are structural elements made of Inconel 625 (9\% Mo included) and a potential contaminant over the Be-coating or cladding on the central column [32]. However, it is probable that this Mo source is also too small. Hence the origin of the Mo source is not yet known. In-vessel inspection or post-mortem analysis of tiles after the current operational campaign may provide clues of the Mo source.

\subsection{Plasma effective charge}

Figure 8 shows a $2.5 \mathrm{MA} / 2.7 \mathrm{~T}$ discharge for comparison of plasma effective charge; during the steady-state NB heating, a large $\mathrm{D}_{2}$ puff was applied With increasing Greenwald density fraction, the ELM frequency increased gradually and finally ELM activities disappeared, showing that the plasma back-transited from an H-mode to an L-mode. From the main plasma, no W lines were observed, even the quasi-continuum emission around $5 \mathrm{~nm}$, whose intensity is usually high in a low electron temperature range, in addition to $\mathrm{W}^{45+}$ line at $6.2 \mathrm{~nm}[10,11]$, was not observed. This qualitatively indicates that the $\mathrm{W}$ density in the core plasma is very low in this discharge. This is due to low $\mathrm{W}$ sputtering at the $\mathrm{W}$ divertor because of low ion impact energy to the $\mathrm{W}$ divertor plates, and also due to high screening efficiency against $\mathrm{W}$ ions by the high density SOL plasma. Hence in this plasma, the contribution of the recombination continuum, which is proportional to the $\mathrm{W}$ density, is negligible, and therefore the plasma effective charge can be directly evaluated from the measured continuum intensity. Figure 8 (d) shows comparison of $Z_{\text {eff }}$ evaluated from the continuum of the first order reflection spectrum at $2.4 \mathrm{keV}$ to that from the second at $4.8 \mathrm{keV}$ taken by the X-ray spectrometer as well as the plasma effective charge from the visible spectrometer. Although $Z_{\text {eff }}$ from the $\mathrm{X}$-ray spectrometer varies from 0.9 to 1.7 , these three measurements are in agreement within $50 \%$. 


\subsection{Quantitative validity}

As described in the above two subsections, comparison of the $\mathrm{W}$ concentration evaluated from the $\mathrm{W}^{46+}$ line and that from the $\mathrm{W}^{45+}$ line shows agreement within $20 \%$, and comparison between the plasma effective charge evaluated from the first order reflection spectrum and that from the second shows also agreement within $50 \%$. These confirm that the quantities evaluated in this work are self-consistent within $20-50 \%$. In addition, comparison between the plasma effective charge evaluated from the X-ray spectrometer is in fair agreement with that from the visible spectroscopy. This indicates that the absolute sensitivity of the X-ray spectrometer, which was calculated as a photon throughput of the whole spectrometer system [9], is also consistent. Therefore, it is likely that the $\mathrm{W}$ concentrations determined from the X-ray spectrometer are also valid within $\pm 50 \%$.

However, in the comparison of the plasma effective charge, the plasma with negligibly low $\mathrm{W}$ concentrations was chosen for the purpose of simplicity in the analysis, in other words, for ignoring contribution of recombination continuum to the measured continuum. Although this choice is suitable for the investigation of the validity of the sensitivity of the X-ray spectrometer, validity on the contribution of the recombination continuum, in turn, is not yet investigated. In plasmas with $\mathrm{W}$ concentrations of $\sim 10^{-5}$, it is often seen that the plasma effective charge evaluated from the X-ray spectrometer tends to be higher than that from the visible spectroscopy by a factor of $\sim 3$. This indicates that the recombination continuum, which is subtracted from the measured continuum to evaluate only bremsstrahlung intensity, is underestimated. One of the possibilities of the underestimation is that the recombination continuum is calculated only from the radiative recombination of $\mathrm{W}$ and Mo ions. In other words, emission due to dielectronic recombination, which is a dominant recombination process in high temperature plasmas such as the plasmas in this study, is ignored. Although the dielectronic recombination is a resonance process in terms of transition energy, it is possible to contribute to the continuum due to huge number of radiative transitions forming quasi-continuum emission. Preliminary calculation of the dielectronic recombination indicates that a number of dielectronic recombination transitions fall into the measured wavelength range. To calculate quantitatively the dielectronic recombination spectrum, dielectronic recombination cross-sections are required. However, careful calculation of the dielectronic recombination cross-sections is very time consuming and is left to future work. As a consequence, the evaluation of the plasma effective charge in this study is limited to plasma discharges with negligibly small W contents.

\section{Summary and Conclusions}

The spectrum taken by the new channel of the upgraded high-resolution X-ray spectrometer was analysed to identify the spectral lines for the purpose of determining 
$\mathrm{W}$ concentrations. The $\mathrm{W}^{45+}$ and $\mathrm{W}^{46+} 3 \mathrm{p}-4 \mathrm{~d}$ inner shell excitation lines in addition to $\mathrm{Mo}^{32+} 2 \mathrm{p}-3 \mathrm{~s}$ lines were identified at a wavelength of $\sim 0.52 \mathrm{~nm}$. From the absolute intensities of the $\mathrm{W}^{46+}$ and $\mathrm{Mo}^{32+}$ lines, $\mathrm{W}$ and Mo concentrations were determined: respectively, $\sim 10^{-5}$ and $\sim 10^{-6}$ range for ELMy H-mode plasmas with a plasma current of 2.0- 2.5 MA, a toroidal magnetic field of $2.7 \mathrm{~T}$ and a neutral beam injection power of 14 - 18 MW. Similar level of Mo concentrations were found from the plasma with the outer strike point on the bulk $\mathrm{W}$ tile, indicating that the $\mathrm{W}$ and Mo multilayer on the CFC outer divertor target was not necessarily a source of Mo ions. For the time being, the Mo source is not yet identified. Comparison of the $\mathrm{W}$ concentration determined from the $\mathrm{W}^{46+}$ line and that from the $\mathrm{W}^{45+}$ line showed agreement within $20 \%$. Comparison between a plasma effective charge, $Z_{\text {eff }}$, determined from the first order reflection spectrum and that from the second order showed also agreement within $\sim 50 \%$, and these agreed with that evaluated from a visible spectroscopy within $\sim 50 \%$. Thus, selfconsistency within the present diagnostic is confirmed, and the absolute sensitivity of the $\mathrm{X}$-ray spectrometer is also confirmed, indicating that the $\mathrm{W}$ concentrations determined from the X-ray spectrometer are also valid.

Comparison of $\mathrm{W}$ concentrations derived by other diagnostics [10,33,34] are ongoing, and results of this work will be presented elsewhere in the near future.

\section{Acknowledgments}

This work has been carried out within the framework of the EUROfusion Consortium and has received funding from the European Union's Horizon 2020 research and innovation programme under grant agreement number 633053. The views and opinions expressed herein do not necessarily reflect those of the European Commission. This work was partly done with the Minerva framework [35]. One of the authors (T.N) would like to express his gratitude for fruitful discussions, in particular, on ion temperature and bremsstrahlung measurement with Drs. C. Giroud and J. Svensson.

\section{References}

[1] M. Merola, F. Escourbiac, R. Raffray, P. Chappuis, T. Hirai and A. Martin, Fusion Engineering and Design 89 (2014) 890.

[2] T. Hirai, F. Escourbiac, S. Carpentier-Chouchana, A. Fedosov, L. Ferrand et al., Fusion Engineering and Design 88 (2013) 1798.

[3] F. Romanelli and J.E. Contributors, Nuclear Fusion 53 (2013) 104002.

[4] G.F. Matthews, Journal of Nuclear Materials 438, Supplement (2013) S2 .

[5] R. Bartiromo, F. Bombarda, R. Giannella, S. Mantovani, L. Panaccione and G. Pizzicaroli, Review of Scientific Instruments 60 (1989) 237.

[6] L.-G. Eriksson, E. Righi and K.-D. Zastrow, Plasma Physics and Controlled Fusion 39 (1997) 27.

[7] J. Rzadkiewicz et al., Nucl. Instrum. Methods in Phys. Research A 720 (2013) 36.

[8] M. Chernyshova, et al., JINST 9 (2014) C03003.

[9] A.E. Shumack, J. Rzadkiewicz, M. Chernyshova, K. Jakubowska, M. Scholz et al., Review of Scientific Instruments 85 (2014). 
[10] T. Pütterich, R. Neu, R. Dux, A.D. Whiteford, M G O'Mullane and the ASDEX Upgrade Team, Plasma Physics and Controlled Fusion 50 (2008) 085016 (27pp).

[11] T. Nakano, N. Asakura, H. Kubo, J. Yanagibayashi and Y. Ueda, Nucl.Fusion 49 (2009) 115024.

[12] T. Nakano and the JT-60 Team, Journal of Nuclear Materials 415 (2011) S327.

[13] A. Kramida, Yu. Ralchenko, J. Reader, and NIST ASD Team (2013). NIST Atomic Spectra Database (ver. 5.1), [Online]. Available: http://physics.nist.gov/asd [2014, June 3]. National Institute of Standards and Technology, Gaithersburg, MD.

[14] G. Kasprowicz, et al., Proc. SPIE, 8008 (2011) 80080J.

[15] K. Pozniak, et al., Proc. SPIE, 8008 (2011) 800808.

[16] L. Frassinetti, M.N.A. Beurskens, R. Scannell, T.H. Osborne, J. Flanagan, M. Kempenaars, M. Maslov, R. Pasqualotto, M. Walsh and J.-E. Contributors, Review of Scientific Instruments $\mathbf{8 3}$ (2012).

[17] G. Magyar, B. Denne-Hinnov et al JET-P(93)43 (1993).

[18] M. F. Gu, Can. J. Phys. 86 (2008) 675.

[19] J. Clementson, P. Beiersdorfer, G. V Brown and M. F. Gu, Phys Scr 81 (2010) 015301.

[20] E. Källne, J. Källne and R.D. Cowan, Phys. Rev. A 27 (1983) 2682.

[21] K.B. Fournier, W.H. Goldstein, et al., May, Phys. Rev. E 53 (1996) 1084.

[22] J. Li, J. Grumer, W. Li, M. Andersson, T. Brage, R. Hutton, P. Jönsson, Y. Yang and Y. Zou, Phys. Rev. A 88 (2013) 013416.

[23] H.P. Summers, JET-IR 06, 1994.

[24] M Mattioli, G. Mazzitelli, K.B. Fournier, M Finkenthal and L. Carraro, Journal of Physics B: Atomic, Molecular and Optical Physics 39 (2006) 4457.

[25] M. Brix, N.C. Hawkes, A. Boboc, V. Drozdov, S.E. Sharapov and J.-E. Contributors, Review of Scientific Instruments $\mathbf{7 9}$ (2008).

[26] T. Fujimoto, Oxford University Press (2004).

[27] Sutherland, Monthly Notices of the Royal Astronomical Society, vol 300, issue 2 (1998).

[28] C. Giround, et al , "Towards baseline operation integrating ITER-relevant core and edge plasma within the constraint of the ITER-like wall at JET", EX/P5-25 25th IAEA Fusion Energy Conference, St Petersburg, Russia (2014).

[29] M. Valisa, et al , "Heavy Impurity Transport in the Core of JET Plasmas", EX/6-1 25th IAEA Fusion Energy Conference, St Petersburg, Russia (2014).

[30] K. Heinola et al., "Fuel retention in JET ITER-Like Wall from Post Mortem Analysis" PSI 2014, Kanazawa, Japan O-10.

[31] C. Ruset, E. Grigore, I. Munteanu, H. Maier, H. Greuner, C. Hopf, V. Phylipps, G. Matthews, JET-EFDA Contributors, Fusion Engineering and Design 84 (2009) 16621665

[32] T. Hirai, J. Linke, et al Phys. Scr. T128 (2007) 166170

[33] T. Pütterich, R. Neu, R. Dux, A.D. Whiteford, M.G. O'Mullane, H.P. Summers and the ASDEX Upgrade Team, "Calculation and experimental test of the cooling factor of tungsten", Nuclear Fusion 50 (2010) 025012.

[34] T. Pütterich, et al., Proc. of the 24th IAEA Fusion Energy Conference 2012, San Diego, US EX/P3-15

[35] J. Svensson and A. Werner, Proceedings IEEE workshop on intelligent signal processing WISP (2007). 
(a)

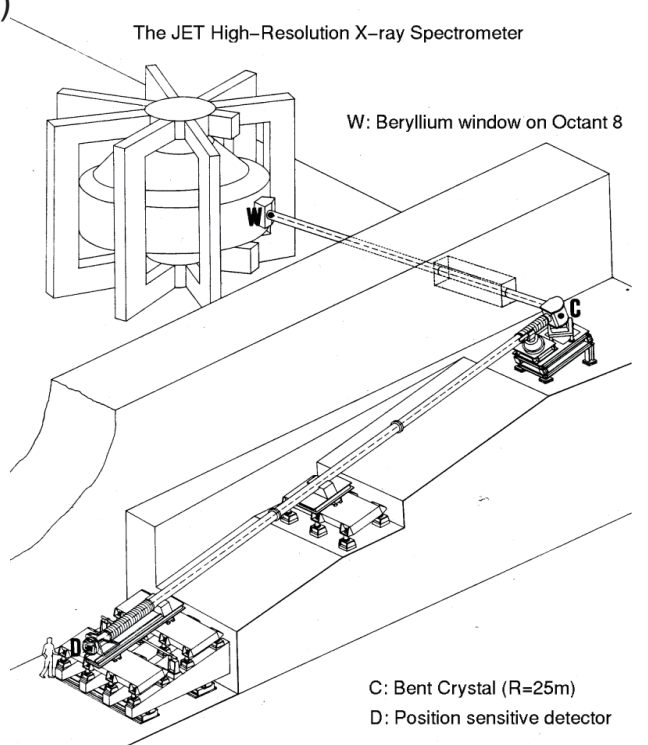

(b)

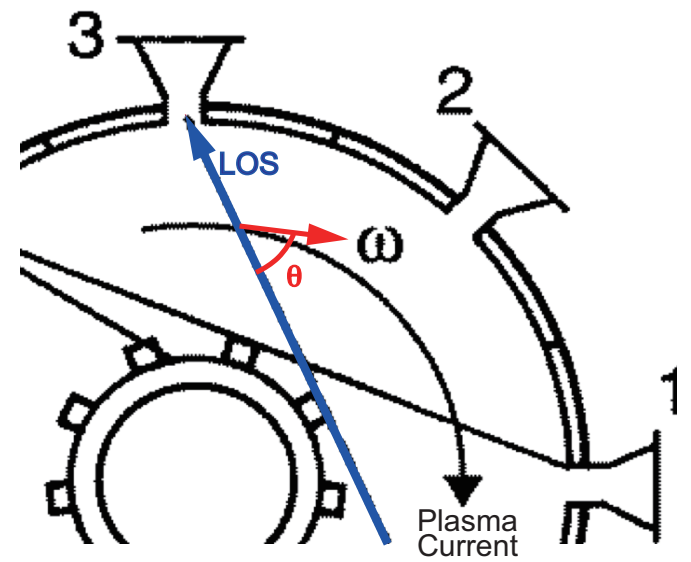

(c)

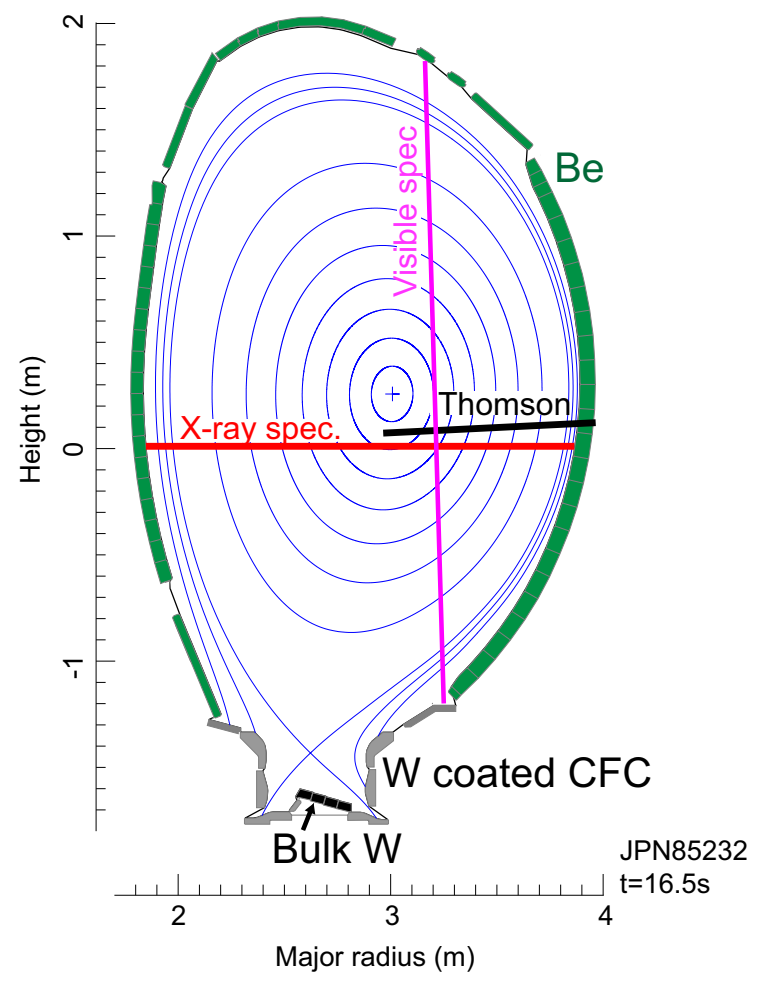

Figure 1. (a) A bird's eye view, (b) line-of-sight on the mid-plane of the torus and (c) line-ofsight on the poloidal cross-section of the X-ray spectrometer [5]. In Fig. (c) line-of-sights of the Thomson scattering system and the visible spectroscopy for bremsstrahlung measurement are also shown. 
Determination of tungsten and molybdenum concentrations from an X-ray range spectrum in JET with th

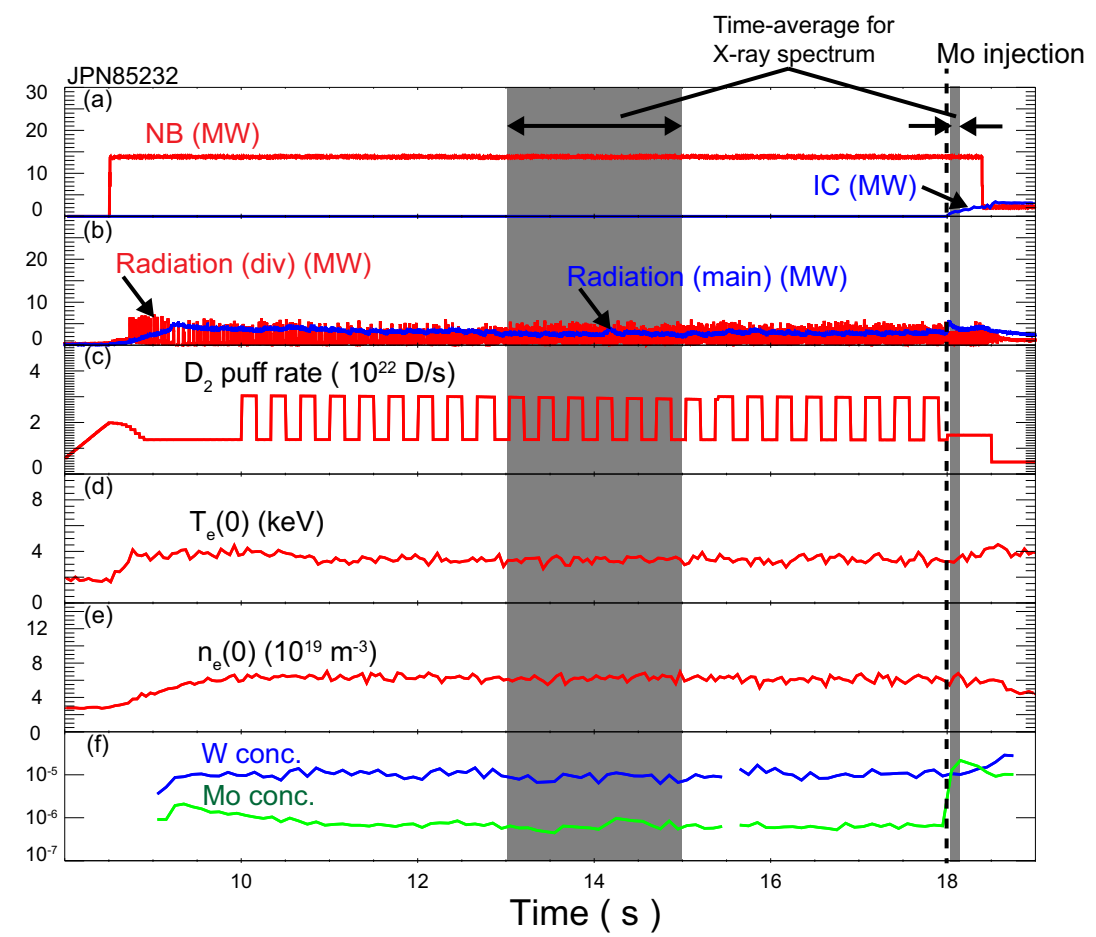

Figure 2. Traces of (a) neutral beam (NB) injection power, ion cyclotron radio frequency (IC) wave injection power, (b) bolometric radiation power from the main plasma, that from the divertor plasma, (c) $D_{2}$ puffing rate, (d) electron temperature and (e) density at the plasma core, and ( $f$ ) tungsten and molybdenum concentrations. 


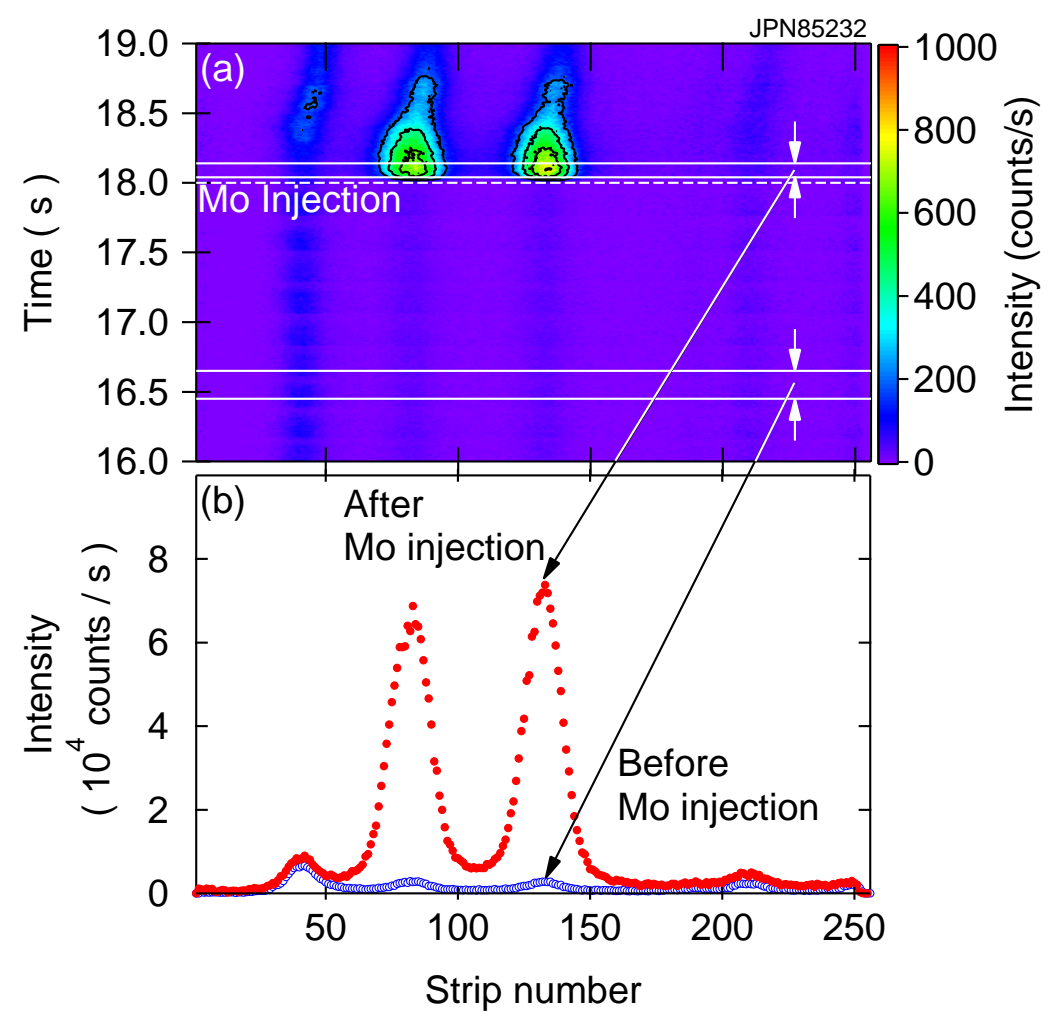

Figure 3. (a) Time evolution of the measured spectrum with Mo injection, and (b) time-averaged spectra before and after the Mo injection. 


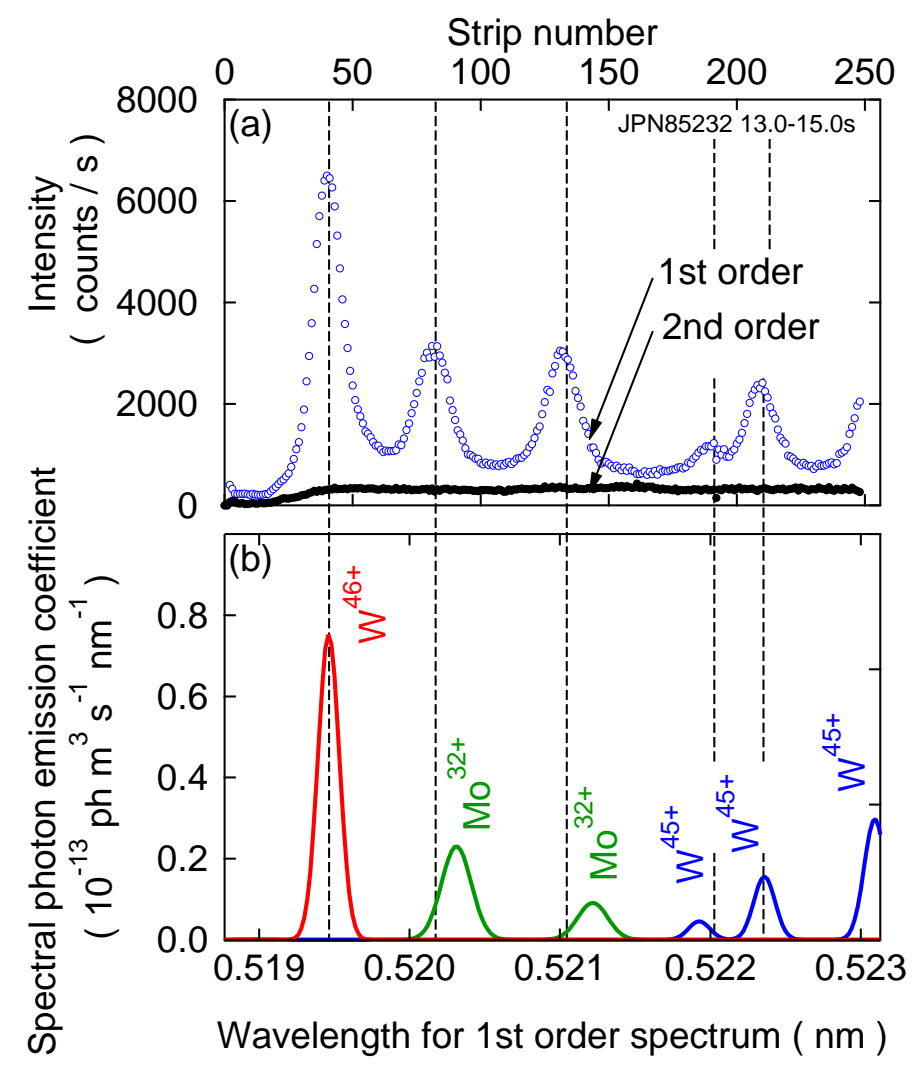

Figure 4. Comparison of the spectrum measured by the upgraded X-ray spectrometer [9] with that calculated by Flexible Atomic Code [18] for $\mathrm{W}^{45+}, \mathrm{W}^{46+}$ and $\mathrm{Mo}^{32+}$ at an electron temperature of $4 \mathrm{keV}$ and a density ratio of 1.0:0.3:0.7, respectively. Note that $W$ and Mo concentration determined from the spectrum are $1 \times 10^{-5}$ and $7 \times 10^{-7}$, respectively. 


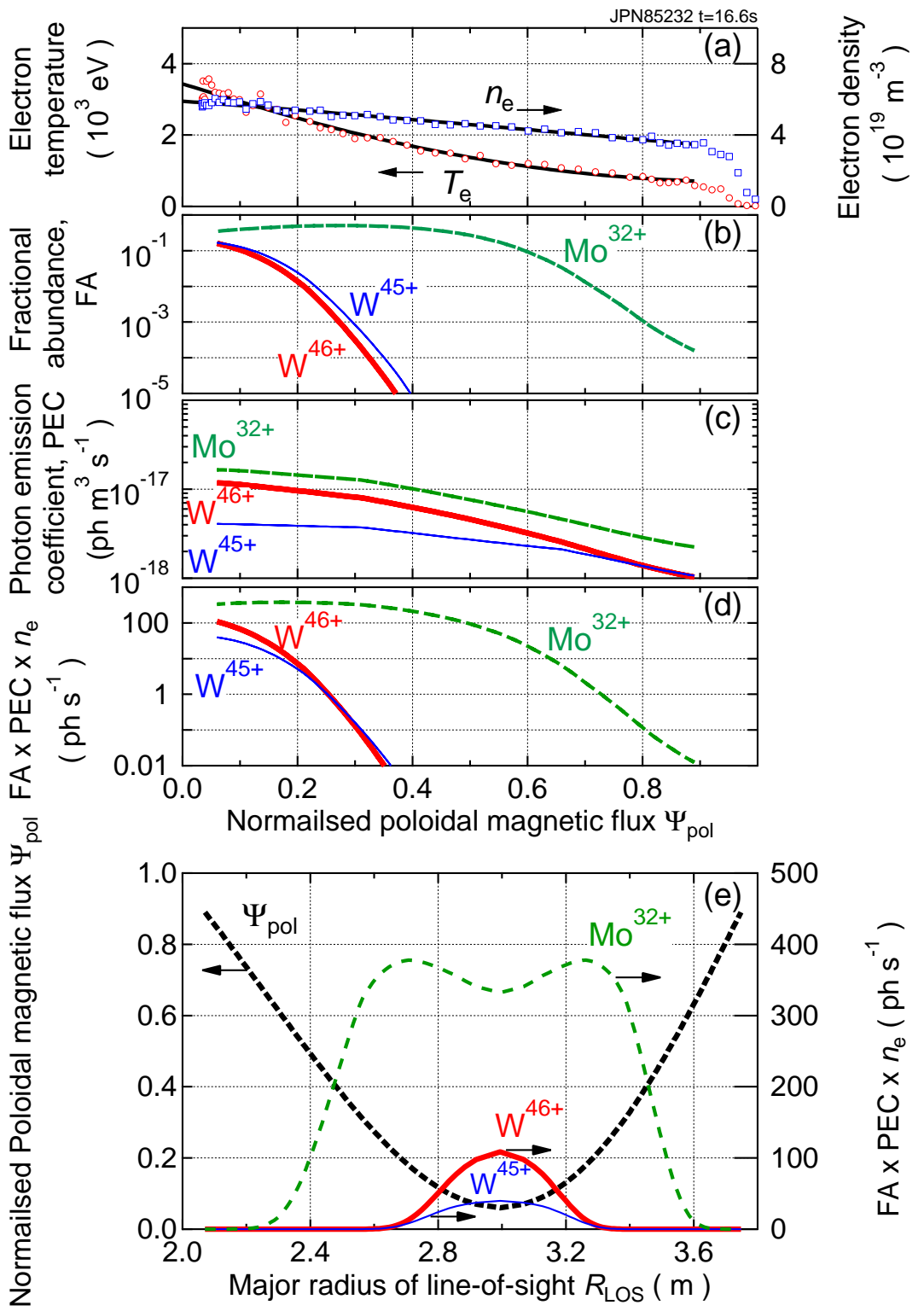

Figure 5. Spatial profiles of (a) measured electron temperature and density, (b) calculated fractional abundances, FA, (c) calculated photon emission coefficients, PEC, (d) products of FA, $P E C$ and $n_{e}$ for $W^{45+}, W^{46+}$ and $M^{32+}$ lines as a function of normalised poloidal magnetic flux, and (e) normalised poloidal magnetic flux and the products of $\mathrm{W}^{45+}, \mathrm{W}^{46+}$ and $\mathrm{Mo}^{32+}$ lines as a function of a major radius of the line-of-sight of the X-ray spectrometer. Note that the left axis of Fig. (e) is a linear scale. 
Determination of tungsten and molybdenum concentrations from an X-ray range spectrum in JET with th

\section{$\underline{\text { Recombination continuum } \quad \text { Bremsstrahlung }}$}

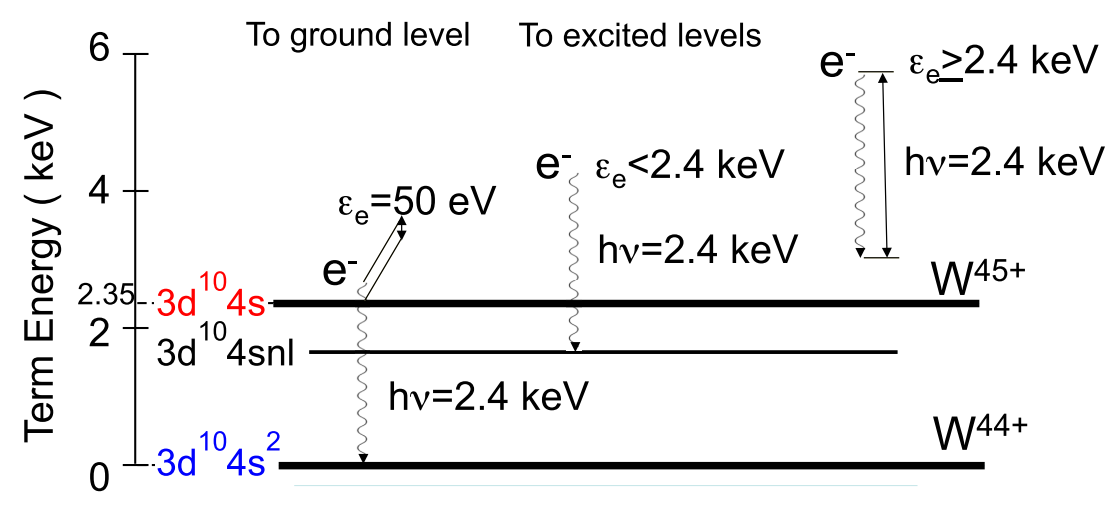

Figure 6. Schematic diagram of energy levels of $\mathrm{W}^{44+}$ and continuum emission processes. $\mathrm{e}^{-}$ indicates a free electron, $\varepsilon_{e}$ a kinetic energy of the free electron, and $\mathrm{h} \nu$ a photon energy.

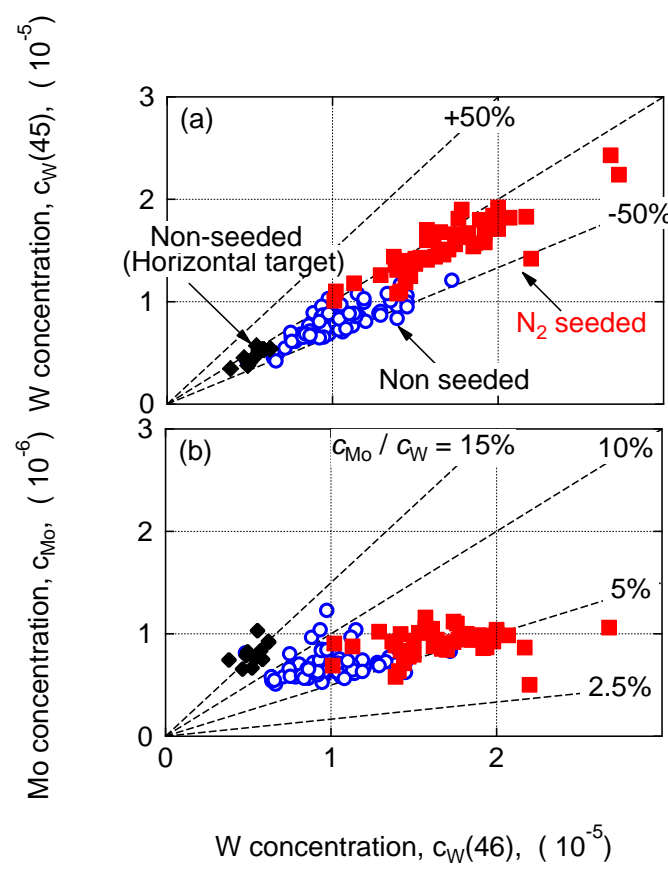

Figure 7. (a) $W$ concentration determined from the $W^{45+} 3 p-4 d$ line, $c_{W}(45)$, and (b) Mo concentration as a function of $W$ concentration determined from the $W^{46+} 3 p-4 d$ line, $c_{W}(46)$, for (blue open circles) a Non-seeded plasma with a plasma current of 2.0 MA, a toroidal magnetic field of $2.7 \mathrm{~T}$ and a neutral beam injection power of $14 \mathrm{MW}$, (red solid squares) an $\mathrm{N}_{2}$ seeded plasma with 2.5 MA, $2.7 \mathrm{~T}$ and $18 \mathrm{MW}$, and (black solid diamonds) a Non-seeded plasma with the outer strike point on the horizontal target (bulk $\mathrm{W}$ target) with $2.5 \mathrm{MA}, 2.7 \mathrm{~T}$ and $17 \mathrm{MW}$. 
Determination of tungsten and molybdenum concentrations from an X-ray range spectrum in JET with th

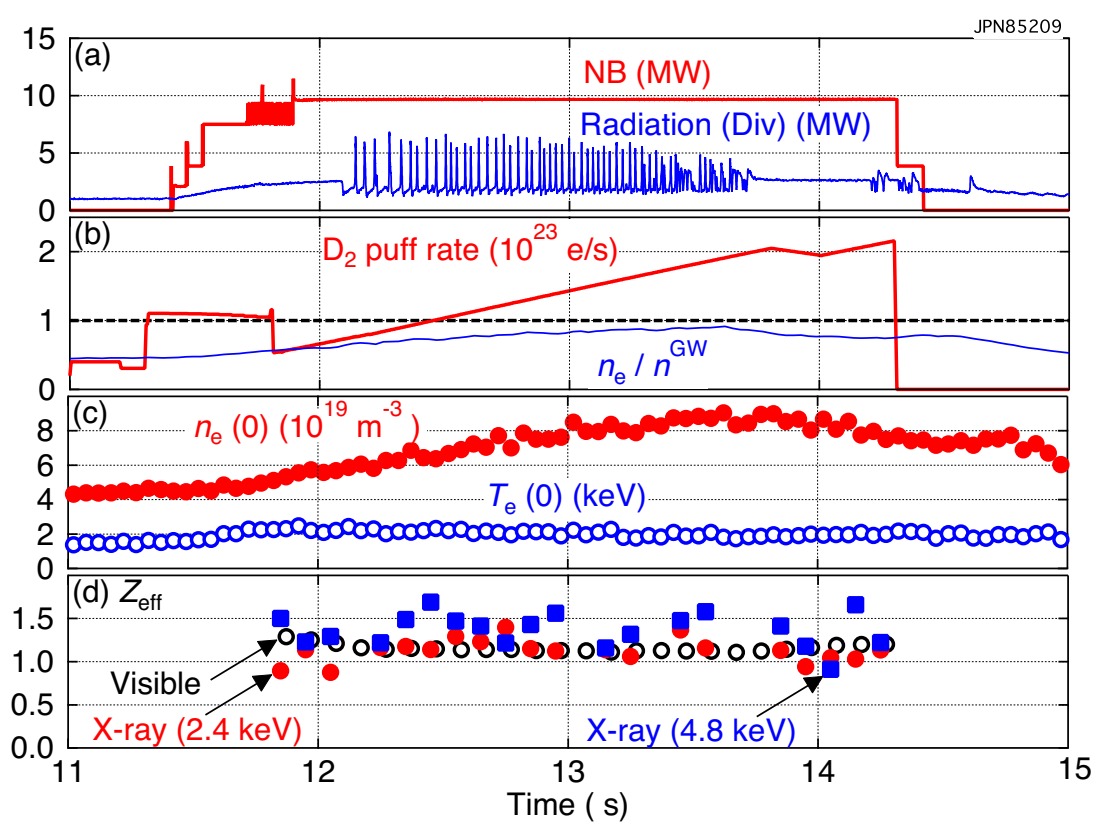

Figure 8. Traces of (a) neutral beam (NB) injection power, bolometric radiation power from the divertor plasma, (b) $D_{2}$ puffing rate, fraction of line-averaged electron density over Greenwald density, (c) electron temperature and density at the plasma core, and (d) plasma effective charge $\left(Z_{\text {eff }}\right)$ determined from a visible spectroscopy and those from the $X$-ray spectrometer $(2.4 \mathrm{keV}$ and $4.8 \mathrm{keV}$ continua), for a plasma with a plasma current of $2.5 \mathrm{MA}$ and a toroidal magnetic field of $2.7 \mathrm{~T}$. 